\title{
Self-Assembled Hyaluronic Acid-Gelatin Microhydrogel for Regenerating Neurite-Like Cells from Induced Pluripotent Stem Cells
}

\author{
Yung-Chih Kuo, Yu-Chun Chen \\ Department of Chemical Engineering, National Chung Cheng University \\ Chia-Yi, Taiwan 62102, Republic of China \\ chmyck@ccu.edu.tw
}

\begin{abstract}
Neuronal differentiation of induced pluripotent stem cells (iPSCs) in repeating particulate units of microhydrogel with hybrid hyaluronic acid (HA) and gelatin (Gel) was investigated. HA and Gel were photocrosslinked, assembled, and further used to regenerate neurons from iPSCs. Stained fluorescent images evidenced that the self-assembled microhydrogel comprising 50\% $\mathrm{HA}$ and $50 \%$ Gel was able to preserved phenotypic iPSCs, leading to an improved production of neuronal lineage after induction with nerve growth factor. The current self-assembled HA-Gel microhydrogel constructs can be properly self-assembled for engineered biomaterials in the duplication of nervous tissue from iPS cells after incubation with neurite-inductive factor.
\end{abstract}

Keywords: Self-assembly; microhydrogel; hyaluronic acid; gelatin; induced pluripotent stem cell; neuron

\section{Introduction}

Gelatin (Gel) and hyaluronic acid (HA) belong to photocrosslinkable biopolymers for various applications in material engineering [1]. Gel is a biodegradable gel, which induces nearly no immunological response for cell binding [2]. In addition, HA containing abundant glycosaminoglycans can stimulate multiplication of cells for wound care and has been use to support the three-dimensional culture for tissue growth [3]. In regard to the regeneration of functional cells for tissue engineering, a designed hydrogel matrix with repeating building blocks from bottom-up technique can be a reasonable method [4].

The purpose of this study is to fabricate self-assembled microhydrogel of HA and Gel to differentiate induced pluripotent stem cells (iPSCs) toward neurons. The structure of self-assembly and the guidance of neuronal differentiation of iPSCs in self-assembled HA-Gel microhydrogel are investigated.

\section{Materials and Methods}

\subsection{Fabrication of HA-Gel microhydrogel}

$10 \%$ (w/v) Gel (Sigma-Aldrich, St. Louis, MO) was dissolved in ultrapure water (Barnstead, Dubuque, IA) at $200 \mathrm{rpm}$ and $60^{\circ} \mathrm{C}$ for $40 \mathrm{~min}$. This Gel solution was reacted with $2.5 \%(\mathrm{v} / \mathrm{v})$ methacrylic anhydride (MA; Sigma-Aldrich) at 700 $\mathrm{rpm}$ and $50^{\circ} \mathrm{C}$ for $2 \mathrm{~h}$ and dialyzed with cellulose bag (Spectrum Laboratories, Rancho Dominguez, CA) of $12 \mathrm{kDa}$ against ultrapure water at $40^{\circ} \mathrm{C}$ for 1 week with a daily replacement of the dialysis buffer, frozen in a low-temperature freezer (Frigidaire, Augusta, GA) at $-20^{\circ} \mathrm{C}$ for $24 \mathrm{~h}$, and freeze dried using a lyophilizer (Eyela, Tokyo, Japan) at $2-4$ torr and $-80^{\circ} \mathrm{C}$ for $24 \mathrm{~h}$. The solid product was preserved at $-20^{\circ} \mathrm{C}$. In addition, $1 \%$ (w/v) HA (Sigma-Aldrich) was dissolved in ultrapure water of $20 \mathrm{~mL}$ at $200 \mathrm{rpm}$ for $20 \mathrm{~min}$. This HA solution was mixed with $4 \%$ (v/v) sodium hydroxide $(\mathrm{NaOH}$; SigmaAldrich) solution of $5 \mathrm{~N}$, reacted with $2.5 \%(\mathrm{v} / \mathrm{v}) \mathrm{MA}$ at $400 \mathrm{rpm}$ for $2 \mathrm{~h}$, stored at $4^{\circ} \mathrm{C}$ for $24 \mathrm{~h}$, mixed with $95 \%$ ethanol (Taiwan Sugar, Tainan, Taiwan) for sediments. After precipitation, HA were washed with $95 \%$ ethanol, placed at $40^{\circ} \mathrm{C}$ for $3 \mathrm{~h}$, dissolved in ultrapure water, frozen at $-20^{\circ} \mathrm{C}$ for $24 \mathrm{~h}$, and freeze dried using the lyophilizer for $24 \mathrm{~h}$. The solid product was preserved at $-20^{\circ} \mathrm{C} .5 \%(\mathrm{v} / \mathrm{v})$ Ocadecyltrichlorosilane (OTS; Sigma-Aldrich) in heptane (Sigma-Aldrich) of of $0.5 \mathrm{~mL}$ was coated on a cover slide of $18 \mathrm{~mm} \times 18 \mathrm{~mm}$. $2.5 \mathrm{~mL}$ of the OTS solution was coated on a cover slide of $76 \mathrm{~mm} \times 22$ $\mathrm{mm}$. The OTS solution on the cover slides was evaporated at room temperature for $30 \mathrm{~min}$ and at $80^{\circ} \mathrm{C}$ for $2 \mathrm{~h}$. The cover slides were rinsed with $95 \%$ ethanol and then with $75 \%$ ethanol. The ratio of HA to Gel was 0:100 and 10:90 for 10\% (w/v) 
HA-Gel microhydrogel, 20:80 and 30:70 for 8\% (w/v) HA-Gel microhydrogel, and 40:60 and 50:50 for 6\% (w/v) HA-Gel microhydrogel. 2\% 2-hydroxy-1-(4-(hydroxyethoxy)phenyl)-2-methyl-1-propanone (Ciba Chemicals, Perth, Western Australia) was mixed with the HA and Gel hydrogel in complete darkness for $1 \mathrm{~h}$. The hydrogel of $20 \mu \mathrm{L}$ was added in the space between two OTS-treated cover slides of $150 \mu \mathrm{m}$ with a photomask on the top and crosslinked with ultraviolet (UV) in a distance of $1.5 \mathrm{~cm}$ at $365 \mathrm{~nm}$ and $4.5 \mathrm{~mW} / \mathrm{cm}^{2}$ for $1 \mathrm{~min}$. The defined pattern on the photomask showed regular squares of $400 \mu \mathrm{m} \times 400 \mu \mathrm{m}$ with a distance of $1 \mathrm{~mm}$ between neighboring squares (Taiwan Kong King Co., Taoyuan, Taiwan). The nontransparent area on the photomask was printed with silver bromide on a sheet of polyethylene terephthalate at a density of $10000 \mathrm{dpi}$. After photocrosslinking, the photomask was removed and the HA-Gel microhydrogel were washed with ultrapure water at $37^{\circ} \mathrm{C}$.

\subsection{Self-assembly of HA-Gel microhydrogel}

HA-Gel microhydrogel was taken from the cover slide and added to mineral oil (Sigma-Aldrich). The noncroslinked hydrogel of $1 \mu \mathrm{L}$ was added around the microhydrogel. The microhydrogel-in-oil system was disturbed with a needle tip. After flocculation, the HA-Gel microhydrogel was exposed to UV again in a distance of $1.5 \mathrm{~cm}$ at $365 \mathrm{~nm}$ and $4.5 \mathrm{~mW} / \mathrm{cm}^{2}$ for $10 \mathrm{~s}$. The optical morphology of HA-Gel microhydrogel self-assembly was visualized using a phase contrast biological microscope (Motic, Richmond, BC, Canada).

\subsection{Staining of iPSCs in self-assembled HA-Gel microhydrogel}

The method for the propagation of iPSCs (System Biosciences, Mountain View, CA) was proposed previously [5]. The expanded iPSCs at a density of $5 \times 10^{5}$ cells $/ \mathrm{mL}$ were added to the mixed hydrogel. The construct iPSC-laden selfassembled microhydrogel was prepared with iPSCs in the mixed hydrogel of $20 \mu \mathrm{L}$ on the OTS-coated cover slide using UV in a distance of $1.5 \mathrm{~cm}$ at $365 \mathrm{~nm}$ and $4.5 \mathrm{~mW} / \mathrm{cm}^{2}$ for $1 \mathrm{~min}$. The cell-laden self-assembled HA-Gel microhydrogel constructs were cultivated with ESGRO complete PLUS clonal grade medium (Millipore, Billerica, MA) containing 1\% penicillin-streptomycin-glutamate solution (Gibco, Carlsbad, CA) in a humidified $\mathrm{CO}_{2}$ incubator (NuAire, Plymouth, MN) at $37^{\circ} \mathrm{C}$ for 1-3 days. In addition, the constructs were cultivated with recombinant human beta-nerve growth factor (NGF; PeproTech, Rocky Hill, NJ) at a concentration of $10 \mathrm{ng} / \mathrm{mL}$ for 3 days. The cultivated constructs were treated with $10 \%$ (v/v) formaldehyde solution (Sigma-Aldrich), desiccated with ethanol of 70\%-99.8\%, immersed in o-xylene, imbedded in paraffin, solidified with ice, and sliced using a paraffin section microtome (Leica, Nussloch, Germany) to samples of $4 \mu \mathrm{m}$. The samples were baked at $56^{\circ} \mathrm{C}$, immersed in o-xylene, rinsed with $99.8 \%$ ethanol, hydrated with ethanol of $95 \%-70 \%$, washed with ultrapure water, boiled in citric acid buffer (J. T. Baker, Phillipsburg, NJ), permeated with $0.5 \%$ (v/v) Triton X100 (Acros Organics, Geel, Belgium), incubated with serum blocking solution (Zymed Laboratories, South San Francisco, CA), reacted with Milli-Mark anti-stage-specific embryonic surface antigen-1 (SSEA-1) cloning MC-480 phycoerythrin (PE) label (1:100, Millipore) and with rabbit monoclonal antibody [EP1331Y] to $\beta$ III tubulin (1:100, Abcam, Cambridge, MA) at $4^{\circ} \mathrm{C}$ overnight, incubated with goat polyclonal antibody to rabbit immunoglobulin $\mathrm{G}$ (heavy and light) with fluorescein isothiocyanate label $\left(1: 100\right.$, Abcam) at $25^{\circ} \mathrm{C}$ in darkness, stained with $0.5 \%$ (w/v) 4',6-diamidino-2-phenylindole (SigmaAldrich) in $0.5 \%(\mathrm{v} / \mathrm{v})$ Triton X-100 at $25^{\circ} \mathrm{C}$ in darkness, dried, and treated with ProLong gold antifade reagent (Biocompare, South San Francisco, CA). The red fluorescence (SSEA-1), green fluorescence (BIII tubulin), and blue fluorescence (nucleus) were visualized using a confocal laser scanning microscope (LSM 510, Zeiss, Oberkochen, Germany) with a filtered argon laser at wavelength for excitation at $555 \mathrm{~nm}, 495 \mathrm{~nm}$, and $364 \mathrm{~nm}$ and emission at $578 \mathrm{~nm}, 528 \mathrm{~nm}$, and 454 nm.

\section{Results and Discussion}

\subsection{Self-assembly of HA-Gel microhydrogel}

Fig. 1 shows the self-assembly of HA-Gel microhydrogel comprising singlet in water and mineral oil. As indicated in Fig. 1(a), discrete HA-Gel microhydrogel attached to each other on water. This was because the hydrophilic attraction was able to coagulate the photocrosslinked HA-Gel microhydrogel. In addition, the structure of the singlet microhydrogel was consistent with the transparent area of the photomask used. As revealed in Fig. 1(b), a close pack of linear configuration was observed. Thus, the hydrophilic interaction could be applied to control the self-assembly of HA-Gel microhydrogel. As 
displayed in Fig. 1(c), a self-assembled aggregate of HA-Gel microhydrogel was observed in oil. This aggregation resulted mainly from the stirring force and affinity among the particulate microhydrogel.

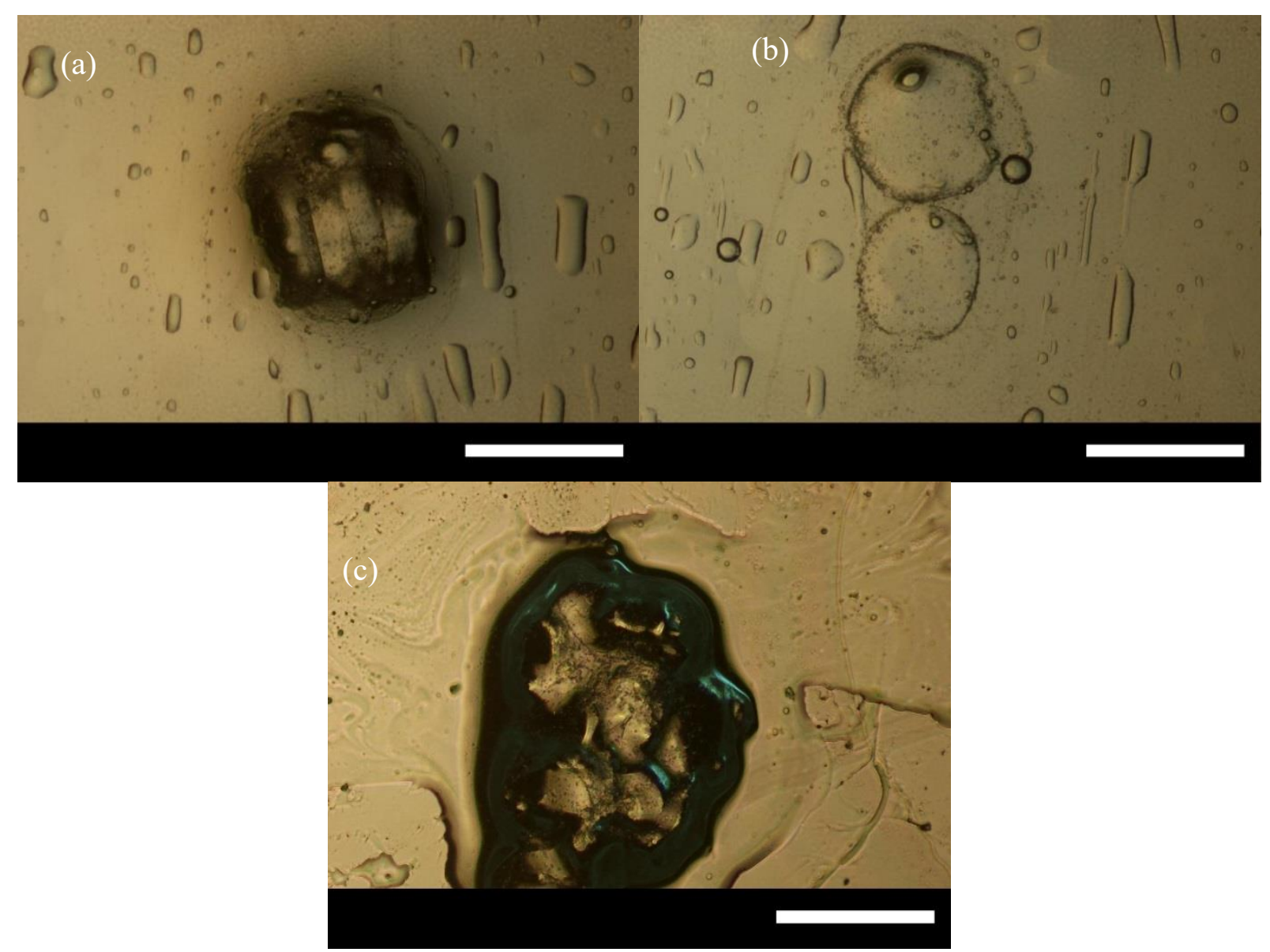

Fig. 1: The morphology of self-assembled microhydrogel. (a) side by side in water, (b) linear coagulation in water, (c) assembly in mineral oil. Bar $=400 \mu \mathrm{m}$.

\subsection{Stained fluorescence of iPSCs in self-assembled HA-Gel microhydrogel}

Fig. 2-4 shows the immunochemical staining images of SSAE-1 (embryonic cell marker) and $\beta I I I$ tubulin (neuronlike cell marker) in iPSC-laden self-assembled HA-Gel microhydrogel. As indicated in Fig. 2, the red dots testified that selfassembled HA-Gel microhydrogel could preserve the phenotype of iPSCs. In addition, an increase in the weight percentage of HA enhanced the red intensity in self-assembled HA-Gel microhydrogel. This was because HA could maintain the pluripotentiality of iPSCs in the embryonic state [10]. As revealed in Fig. 3, the red signals decreased from those shown in Fig. 2. However, a portion of cells still bore SSEA-1. This was because Gel contained abundant functional groups such as $\mathrm{S}-\mathrm{H}$ and $\mathrm{NH}_{2}$, trigger the differentiation of iPSCs [6]. Moreover, the medium used for induction was quite different from that used for multiplying iPSCs. The basic medium included leukemia inhibitory factor, while the induction medium resulted in the sensitivity of iPSCs to their neighboring molecules [7]. As displayed in Fig. 3, after an induction with NGF, the preserved iPSCs in self-assembled HA-Gel microhydrogel differentiated toward $\beta I I I$ tubulin-positive cells and the embryonic signals were extremely feeble. Based on the very few iPSCs identified by anti-SSEA-1, it could be concluded that NGF was effective in the production of neurons from the preserved SSEA-1-possitive cells in self-assembled HA-Gel microhydrogel. An increase in the weight percentage of HA also intensified the green signals. This demonstrated that the phenotypic iPSCs could be first stabilized and then regenerated the neural linage cells in self-assembled HA-Gel microhydrogel. Furthermore, in in the case of an incorporated induction factor, self-assembled HA-Gel microhydrogel with high HA level reduced the influence of Gel on the phenotype of iPSCs. 
(a)

(b)

(c)

(d)

(e) (f)

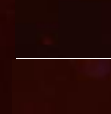

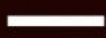

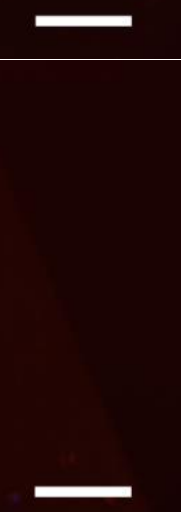

Fig. 2: Immunofluorescent staining of cultured iPSC-laden microhydrogel for 1 day. (a) 10\% HAMA:GelMA=0:100, (b) 10\% HAMA:GelMA=10:90, (c) 8\% HAMA:GelMA=20:80, (d) 8\% HAMA:GelMA=30:70, (e) 6\% HAMA:GelMA=40:60, (f) 6\% HAMA:GelMA $=50: 50$. Bar $=60 \mu \mathrm{m}$.

(a)

(d) (b)

(e) (c)

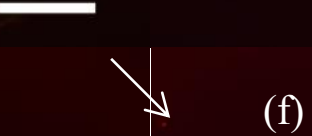

Fig. 3: Immunofluorescent staining of cultured iPSC-laden microhydrogel for 3 days. (a) 10\% HAMA:GelMA=0:100, (b) 10\% HAMA:GelMA=10:90, (c) 8\% HAMA:GelMA=20:80, (d) 8\% HAMA:GelMA=30:70, (e) 6\% HAMA:GelMA=40:60, (f) 6\% HAMA:GelMA $=50: 50$. Bar $=120 \mu \mathrm{m}$. 


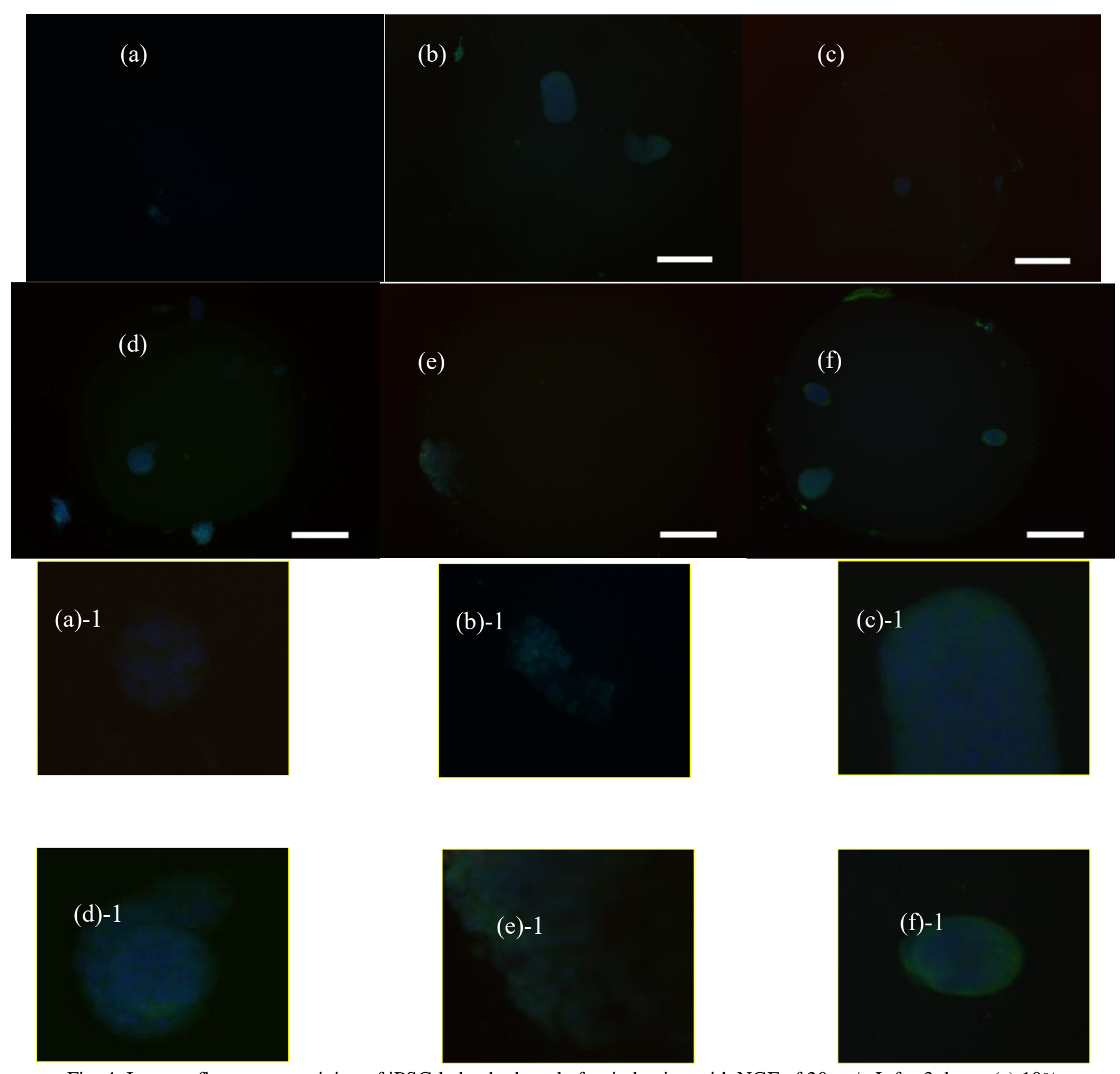

Fig. 4: Immunofluorescent staining of iPSC-laden hydrogel after induction with NGF of $20 \mathrm{ng} / \mathrm{mL}$ for 3 days. (a) $10 \%$ HAMA:GelMA=0:100, (b) 10\% HAMA:GelMA=10:90, (c) 8\% HAMA:GelMA=20:80, (d) 8\% HAMA:GelMA=30:70, (e) 6\% HAMA:GelMA=40:60, (f) 6\% HAMA:GelMA=50:50. (a-1), (b-1), (c-1), (d-1), (e-1), (f-1) magnified images. Bar = $120 \mu$ m.

\section{Conclusion}

Neuronal regeneration in self-assembled HA-Gel microhydrogel from iPSCs was performed. The stirring of photocrosslinked HA-Gel microhydrogel in mineral oil yielded a linear self-assembly. The self-assembled HA-Gel microhydrogel could preserve the phenotype of iPSCs in the cell-laden constructs. In addition, NGF activated the differentiation of iPSCs toward neurons in self-assembled HA-Gel microhydrogel. An induction of iPSCs with NGF in selfassembled HA-Gel microhydrogel with a high weight percentage of HA can be promising to reframe the nerve tissue for clinical application. 


\section{Acknowledgements}

This work is supported by the Ministry of Science and Technology of the Republic of China.

\section{References}

[1] J. L. Ifkovits and J. A. Burdick, "Review: photopolymerizable and degradable biomaterials for tissue engineering applications," Tissue Eng., vol. 13, pp. 2369-2385, 2007.

[2] M. A. Horton, "The $\alpha v \beta 3$ integrin "vitronectin receptor," Int. J. Biochem. Cell. Biol., vol. 29, pp. 721-725, 1997.

[3] S. H. Hsu, S. W. Whu, S. C. Hsieh, C. L. Tsai, D. C. Chen, and T. S. Tan, "Evaluation of chitosan-alginate-hyaluronate complexes modified by an RGD-containing protein as tissue-engineering scaffolds for cartilage regeneration," Artif. Organs, vol. 28, pp. 693-703, 2004.

[4] L. Yeh, Y. Ling, J. M. Karp, J. Gantz, A. Chandawarkar, G. Eng, J. Blumling III, R. Langer, and A. Khakemhosseini, "Micromolding of shape-controlled, harvestable cell-laden hydrogels," Biomaterials, vol. 27, pp. 5391-5398, 2006.

[5] Y. C. Kuo and M. J. Huang, "Material-driven differentiation of induced pluripotent stem cells in neuron growth factorgrafted poly( $\varepsilon$-caprolactone)-poly( $\beta$-hydroxybutyrate) scaffolds," Biomaterials, vol. 33, pp. 5672-5682, 2012.

[6] J. M. Curran, R. Chen, and J. A. Hunt, "The guidance of human mesenchymal stem cell differentiation in vitro by controlled modifications to the cell substrate," Biomaterials, vol. 27, pp. 4783-4793, 2006.

[7] Y. C. Kuo, C. F. Yeh, and J. T. Yang, "Differentiation of bone marrow stromal cells in poly(lactide-coglycolide)/chitosan scaffolds," Biomaterials, vol. 30, pp. 6604-6613, 2009. 